Matthias Berg, Olaf Blaschke, Martin Sabrow, Jens Thiel, Krijn Thijs, Die versammelte Zunft. Historikerverband und Historikertage in Deutschland 1893-2000

\title{
Antonin Dubois
}

\section{OpenEdition}

Journals

Édition électronique

URL : http://journals.openedition.org/ifha/10304

DOI : 10.4000/ifha.10304

ISSN : 2198-8943

Éditeur

IFRA - Institut franco-allemand (sciences historiques et sociales)

\section{Référence électronique}

Antonin Dubois, « Matthias Berg, Olaf Blaschke, Martin Sabrow, Jens Thiel, Krijn Thijs, Die versammelte Zunft. Historikerverband und Historikertage in Deutschland 1893-2000", Revue de I'IFHA [En ligne], Date de recension, mis en ligne le 20 août 2019, consulté le 24 septembre 2020. URL : http:// journals.openedition.org/ifha/10304; DOI : https://doi.org/10.4000/ifha.10304

Ce document a été généré automatiquement le 24 septembre 2020.

(C)IFHA 
Matthias Berg, Olaf Blaschke, Martin Sabrow, Jens Thiel, Krijn Thijs, Die versammelte Zunft. Historikerverband und Historikertage in Deutschland 1893-2000

Antonin Dubois

\section{RÉFÉRENCE}

Matthias Berg, Olaf Blaschke, Martin Sabrow, Jens Thiel, Krijn Thijs, Die versammelte Zunft. Historikerverband und Historikertage in Deutschland 1893-2000, Göttingen : Wallstein Verlag, 2018, 839 p., 39,90€ 
Il existe en France plusieurs associations d'historiennes et historiens professionnels, comme la Société des historiens médiévistes de l'enseignement supérieur public ou l'Association des historiens modernistes des universités françaises, ou encore des associations qui ont pour but de défendre la recherche historique scientifique, ainsi le Comité de vigilance face aux usages publics de l'histoire. Il n'existe par contre pas d'association unique représentant l'ensemble de la corporation historienne, ce qui s'explique notamment par la forte centralisation du champ universitaire français. C'est en revanche le cas en Allemagne, et ce depuis bientôt 125 ans, malgré plusieurs interruptions, avec le Verband der Historiker und Historikerinnen Deutschlands (VHD), nom officiel depuis

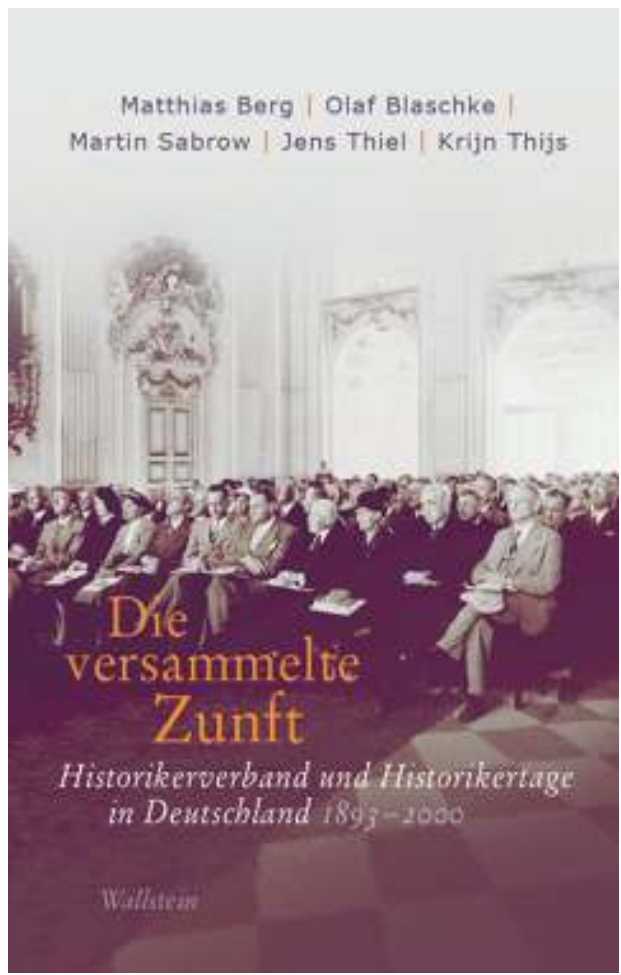
1998 du Verband deutscher Historiker fondé en 1895 .

L'histoire de la profession historique est depuis de nombreuses années l'objet d'études en Allemagne (voir, parmi les plus importantes, sur les deux dictatures du $\mathrm{XX}^{\mathrm{e}}$ siècle : Winfried Schulze et Otto Gerhard Oexle, Deutsche Historiker im Nationalsozialismus, 1999 et Karl Heinrich Pohl, Historiker in der DDR, 1997). Une histoire complète du VHD était en projet depuis 2011. Ce livre, rédigé à dix mains pour un total de plus de 800 pages, en est l'aboutissement, après plusieurs publications intermédiaires (notamment le numéro spécial de la revue Geschichte in Wissenschaft und Unterricht, vol. 64, n 3-4, 2013).

L'objet de cet ouvrage est d'analyser « les conditions, les possibilités et les problèmes d'institutionnalisations ou d'institutions dans les sciences historiques » (p.12). À travers l'histoire du VHD et de son pendant socialiste sous la République démocratique allemande, c'est donc une histoire de la discipline historique que souhaitent mener les auteurs. Ils proposent pour cela six perspectives : le rôle des associations dans la professionnalisation du métier d'historien et dans la standardisation de la science historique, les formes des rencontres corporatives publiques (les congrès dans leurs aspects scientifiques et de sociabilité), la place et l'ambition institutionnelles des associations, l'articulation entre le national et l'international avec le comité et les congrès internationaux d'historiens, les relations des associations avec l'extérieur, en particulier les champs scientifique et politique et le débat public (p. 14-19).

Les chapitres sont divisés chronologiquement et suivent eux-mêmes une structure chronologico-thématique. Matthias Berg a pris en charge la rédaction des cinq premiers, c'est-à-dire l'ensemble du premier volume (à l'exception de l'introduction générale), consacré à la période allant du premier congrès national des historiens en 1893 à la fondation des deux États allemands en 1949.

En effet, l'histoire du VHD est inséparable de celle des congrès nationaux, les Historikertage. Le premier d'entre eux est organisé à Munich en 1893. Des conférences 
scientifiques y sont prononcées, mais l'un des principaux objets du congrès est le contenu et la réforme de l'enseignement de l'histoire au Gymnasium, que l'empereur Guillaume II entend infléchir dans un sens patriotique. Un deuxième congrès est organisé à Leipzig l'année suivante, puis un troisième à Francfort-sur-le-Main en 1895, lors duquel l'association Verband deutscher Historiker est créée. Son but principal est d'assurer l'organisation et la bonne tenue de ces congrès nationaux à échéance régulière. Dix autres congrès ont lieu avant le déclenchement de la Première Guerre mondiale, dont deux en Autriche (Innsbruck, 1896 et Vienne, 1913). La guerre cause une interruption de onze ans et ce n'est qu'en 1924 qu'un nouveau congrès peut se tenir, à nouveau à Francfort, la crise de la Ruhr ayant forcé l'annulation du congrès qui devait avoir lieu à Cologne l'année précédente. Les historiens allemands arrivent à se réunir quatre autres fois durant la République de Weimar, dont une en Autriche (Graz, 1927).

Le premier volume présente de manière très détaillée les discussions et les débats qui agitent les historiens entre chaque congrès - l'organisation de chacun d'entre eux étant toujours sujette à polémiques et à problèmes. Cette exhaustivité impose des longueurs et cette multitude de détails et d'anecdotes empêche parfois la bonne compréhension des éléments essentiels à l'histoire du VHD. Néanmoins, elle témoigne bien de la précarité générale - malgré quelques années fastes - de l'association durant son premier demi-siècle. Le VHD souffre presque continuellement de problèmes logistiques et d'un manque d'investissement de la part de nombreux historiens : plusieurs refusent d'adhérer à l'association, surtout avant la Grande Guerre; beaucoup de membres ne payent pas leur cotisation; des responsables de l'organisation d'un congrès ne s'occupent pas des tâches qui leur incombent voire abandonnent leur mission. Cela devient particulièrement clair lorsqu'est raconté l'échec de l'organisation d'un congrès en 1934 (p. 262 et suivantes). L'arrivée au pouvoir des nazis pose des problèmes spécifiques supplémentaires, qui rendent l'organisation d'un congrès encore plus indispensable que lors des décennies précédentes, car l'existence même du VHD est menacée par la politique de Gleichschaltung, puis plus généralement par la politique scientifique nationale-socialiste. Ce n'est que quelques mois après l'accession à la présidence de l'historien et recteur de l'université de Francfort-sur-le-Main Walter Platzhoff, qui était également entre autres «membre bienfaiteur» de la SS, en 1937, qu'une partie des historiens allemands peuvent se réunir publiquement - à l'exclusion des «non-aryens ». Le début de la guerre met ensuite fin de facto aux activités de l'association, nationales comme internationales.

Dès 1946, des historiens de différentes générations prennent l'initiative d'organiser une réunion informelle pour refonder le VHD. Les ambitions de refonder l'association des historiens allemands aboutit officiellement en 1949 avec la tenue d'un congrès à Munich. La capitale bavaroise a été choisie non seulement du fait du symbole (c'est à Munich que le premier congrès des historiens a été organisé, 56 ans plus tôt), mais aussi pour des raisons scientifiques et pratiques: Munich est l'un des principaux centres d'histoire en Allemagne et l'une des grandes villes universitaires les moins touchées par les bombardements durant la guerre.

Le second volume débute avec ce nouveau congrès munichois et parcourt la seconde moitié du XXe siècle, des deux côtés du mur. En effet, tandis que le VHD est refondé à l'Ouest, une association concurrente, la Historiker-Gesellschaft, est constituée en RDA en 1958. 
Comme le montrent Olaf Blaschke et Jens Thiel, la reconstitution du VHD s'accompagne d'une révision des statuts qui conduit à un renforcement des structures de l'association afin de lui offrir une plus grande stabilité et d'assurer un suivi plus certain et une meilleure régularité dans ses activités, notamment l'organisation des congrès. Durant le premier demi-siècle de l'association, trop souvent les membres des comités locaux ont délaissé l'organisation du congrès dont ils étaient chargés, ce qui a conduit à de nombreux reports et annulations. Pour éviter ce problème, il est décidé que le comité directeur prenne en charge une large part de l'organisation, notamment de la partie scientifique (séances, communications). Cela permettait, surtout sous la présidence du conservateur (tant d'un point de vue politique qu'historiographique) Gerhard Ritter, de choisir qui pouvait communiquer et de limiter le nombre d'intervenants aux méthodes déviant trop de l'historisme dominant. Néanmoins, l'histoire sociale est progressivement mieux acceptée durant les années 1960/70. Deux de ses principaux défenseurs, mais qui sont considérés comme occupant une position modérée par rapport à la jeune génération d'historiens du social, prennent la tête du VHD, Werner Conze (1972-1976) et Gerhard A. Ritter (1976-1980), ce dernier ne devant pas être confondu avec son prédécesseur et presque homonyme. Surtout, le succès est au rendez-vous dès les années 1950. En une décennie, entre le congrès de refondation à Munich en 1949 et le cinquième congrès d'après-guerre à Trier en 1958, le nombre de participants inscrits au Historikertag double. Entre ces deux congrès, le nombre de communications a quant à lui triplé, passant de 10 à 30 (p. 385).

La reconstitution du VHD s'accompagne également d'une professionnalisation : tandis que des enseignants du secondaire et surtout des archivistes avaient jusque-là joué un rôle important au sein de l'association, seuls des historiens universitaires participent au comité de refondation du VHD. Si aucun de ces historiens n'a occupé une position politique de premier rang sous le nazisme, plusieurs d'entre eux avaient des sympathies pour le parti au pouvoir et avaient même parfois soutenu plus ou moins ouvertement le régime hitlérien et ses idées, comme le grand promoteur de la Ostforschung Hermann Aubin, qui devient néanmoins président du VHD entre 1953 et 1958. La tolérance des historiens allemands avec l'expérience nationale-socialiste en général et avec leurs collègues au passé nazi en particulier s'exprime doublement dès la seconde moitié des années 1940. Premièrement, des dizaines de textes sont publiés dès le lendemain de la guerre, cherchant à attaquer la thèse du Sonderweg et présentant le nazisme non pas comme une spécificité allemande, mais comme une erreur, une déviation, un accident dans l'histoire allemande. Deuxièmement, les historiens cherchent à se dédouaner réciproquement (à l'exception des plus ouvertement et clairement engagés dans la machine hitlérienne) auprès des commissions de dénazification. Dans ces deux activités, l'historien national-conservateur, mais qui avait gardé ses distances avec le nazisme, Gerhard Ritter joue un rôle important, légitimé par sa position dominante au sein du monde académique en tant qu'un des principaux acteurs de la refondation du VHD et son premier président d'après-guerre. Au sein du VHD, comme dans la discipline historique, les autres professions, les Églises, la politique et la société en général, c'est donc une politique de réintégration de tous ceux pas ou peu inquiétés par les mesures de dénazification qui est très rapidement adoptée. Cette position, selon laquelle les Allemands sont plus victimes que coupables du nazisme, est largement partagée dans le milieu historien et prévaut jusqu'à la fin des années 1960. Ce n'est que lors du congrès de 1970 (le premier après la mort de Ritter), qu'une section est consacrée au national-socialisme. 
Cette position, que la guerre froide et l'anticommunisme général dans le milieu historien n'ont fait que renforcer, se retrouve également dans la politique d'adhésion du VHD aux historiens de la zone d'occupation soviétique puis de la RDA durant les années 1950. Les membres du VHD sont favorables à l'ouverture de leur association à leurs collègues qualifiés de «bourgeois » par le pouvoir politique est-allemand. Au contraire, ils rejettent les historiens marxistes-léninistes, mais ne peuvent refuser leur adhésion au VHD et leur participation aux Historikertage - dans la mesure où les autorités est-allemandes leur fournissent une autorisation de quitter le territoire, puisque tous les congrès ont lieu à l'Ouest. Néanmoins, la situation se dégrade au cours des années 1950 et la division est actée en mars 1958 par la création d'une société concurrente en RDA, la Deutsche Historiker-Gesellschaft (DHG).

Six années ont été nécessaires à la création de cette société. Le projet remonte à une décision prise au troisième congrès de la SED, le parti unique de la RDA, en 1952 visant au développement d'une science historique socialiste. C'est d'ailleurs au niveau politique que la création de la DHG a été prise en mains et imposée aux historiens. Il n'est donc pas possible de séparer le versant politique du versant scientifique de cette nouvelle société, créée à la fois pour canaliser le travail des historiens est-allemands et pour concurrencer le VHD. Entre le milieu des années 1960 et 1989, la DHG gagne en importance et chacun de ses congrès attire plus de visiteurs que le précédent. Après la chute du mur, les dirigeants de la DHG cherchent à la fois à réformer leur société et à défendre le bilan historiographique est-allemand. Néanmoins, la DHG ne survit pas aux transformations politiques de l'effondrement du bloc soviétique et l'association est dissoute par le comité directeur le 27 octobre 1990, moins d'un mois après la réunification.

La concentration sur les institutions trouve peut-être dans le chapitre consacré à la DHG ses plus grandes limites. L'auteur, Martin Sabrow, traite presque exclusivement des aspects politico-administratifs de l'histoire de la DHG (création de la société, instances de contrôle, organisation des congrès et autres activités) et adopte un ton beaucoup plus critique que ses co-auteurs. Dès lors, il laisse dans l'ombre des évolutions qui témoignent que, malgré l'indéniable dépendance de la science historique estallemande envers le pouvoir politique, tous les travaux menés en RDA ne sont pas de la simple vulgate marxiste appliquée à des événements historiques. C'est ce qu'on peut voir par exemple avec les recherches sur la résistance allemande au nazisme, sujet hautement politique s'il en est. Les historiennes et historiens est-allemands ont bien plus tôt montré la diversité des groupes de résistants que leurs homologues de l'Ouest, qui jusque dans les années 1970 refusaient cette qualification à tous ceux n'appartenant pas au camp national-conservateur, dont les protagonistes les plus connus sont Claus von Stauffenberg et les autres perpétrateurs de l'attentat contre Hitler du 20 juillet 1944. Si l'objectif des historiens de RDA était d'abord de glorifier la résistance communiste, ils se sont progressivement intéressés aux groupes sociaux-démocrates, aux émigrés et même aux nationaux-conservateurs, dont ils ne nient pas le rôle.

Cette mise de côté de la production historiographique est-allemande tranche avec le contenu des deux chapitres suivants, dans lesquels Olaf Blaschke et Jens Thiel intègrent l'histoire du VHD et de ses congrès, de la fin des années 1950 à 1990, à celle des évolutions historiographiques, notamment sur l'établissement de l'histoire sociale et sur la violente controverse autour du livre de Fritz Fischer sur les buts de guerre de l'Allemagne impériale en 1914 dans le chapitre 8 ou sur les nouveaux courants de 
l'histoire des femmes, du quotidien et des mentalités au chapitre 9. Dans ces deux chapitres, la prise en compte - et même en partie la centralité - dans la démonstration des réflexions (supposée "crise» de l'histoire, rôle de l'histoire dans la société, didactique), débats (histoire sociale versus historisme, Historikerstreit) et problèmes (baisse du nombre d'étudiants en histoire, réforme des programmes scolaires, concurrence de la sociologie et de la science politique) qui agitaient les historiens durant ces décennies, apporte une réelle dynamique et une profondeur à un ouvrage sinon surtout descriptif.

Si la production historiographique est donc inégalement analysée dans l'ouvrage, les auteurs étudient pour l'ensemble de la période l'internationalisation de la discipline, et notamment les formes d'expressions publiques de celle-ci. Les premiers congrès internationaux d'historiens sont organisés au tournant des XIX ${ }^{\mathrm{e}}-\mathrm{XX}^{\mathrm{e}}$ siècles, c'est-àdire au même moment où les historiens allemands mettent en place leur association et leurs propres congrès. Un premier se tient à La Haye en 1898 mais regroupe surtout des diplomates et des hommes politiques. Le premier congrès international officiel des historiens a lieu à Paris en 1900, et est suivi d'un autre à Rome en 1903. Les historiens allemands adoptent d'abord une position mitigée voire de distanciation vis-à-vis de ces congrès internationaux. C'est néanmoins à Berlin que se tient le troisième congrès en 1908. Peut-être de manière encore plus appuyée et ostensible que dans d'autres disciplines, les Allemands sont exclus de la scène historique internationale après la Première Guerre mondiale. Ils ne participent donc pas à la création du Comité international des sciences historiques (CISH) en 1926, mais y sont admis rapidement, ce qui permet leur retour dans les relations scientifiques et leur participation au congrès international d'Oslo en 1928. La seconde moitié du XXe siècle est une période de renforcement rapide des influences et des réseaux internationaux. Contrairement à l'après-Première Guerre mondiale, les vainqueurs de la Seconde décident de ne pas exclure les Allemands des relations scientifiques internationales. Les historiens allemands peuvent participer au premier congrès international organisé depuis la fin du conflit, qui se tient à Paris en 1950, et le VHD est réintégré dès l'année suivante au CISH. En 1955, Gerhard Ritter devient le premier Allemand membre du bureau du CISH, puis son vice-président en 1962. En 1975, Karl Dietrich Edmann est élu président du Comité. À cette intégration dans l'institution officielle s'ajoutent d'autres formes d'investissement international, par exemple les premières rencontres francoallemandes dans la zone d'occupation française dès la fin des années 1940, puis à partir des années 1950 la (re)création des Instituts historiques allemands de Rome (1953) et de Paris (1958/1964).

La fin de l'ouvrage est consacrée aux années 1989/90 puis à la décennie suivante, qui ont été des années de profonds changements également pour la corporation historienne. En janvier 1990, deux jeunes historiens de Berlin-Est appellent à la constitution d'une nouvelle société d'historiens, en opposition au régime et à la production historique officielle représentée par la Historiker-Gesellschaft. Le Unabhängiger Historiker-Verband (UHV), auquel est consacré le chapitre 10 rédigé par Krijn Thijs, est officiellement constitué en avril. Les changements politiques permettent un rapprochement progressif entre historiens est- et ouest-allemands, malgré l'attitude précautionneuse bien qu'intéressée de la direction du VHD. Ce rapprochement est concrétisé au Historikertag de Bochum fin septembre 1990, quelques jours avant la réunification, auquel participent de nombreux historiens de RDA. Le UHV participe activement au débat public du début des années 1990, et milite notamment en faveur 
d'un accès illimité aux archives de la Stasi pour les chercheurs. Très rapidement après la réunification, les instituts est-allemands sont "évalués " par des historiens de l'Ouest, sous la houlette du VDH, et partiellement intégrés dans le système de la RFA. Stefan Wolle et Armin Mitter, les deux fondateurs du UHV, critiquent fortement le maintien en poste d'un certain nombre de personnes ayant activement participé au régime dictatorial de la SED, notamment d'enseignants ayant servi d'informateurs auprès de la Stasi contre des étudiants. L'éloignement de Mitter au milieu des années 1990 du monde universitaire témoigne de la difficile intégration des jeunes historiens d'ex-RDA dans le système ouest-allemand. Le faible nombre d'adhérents au VHD provenant des cinq nouveaux Bundesländer au cours des années 1990 en est un autre témoignage.

Les années 1990 sont également pour le VHD celles d'une forte augmentation du nombre de participants et de sections organisées au Historikertag: entre 2000 et 3000 personnes y prennent part, chiffre qui augmente encore à partir du début des années 2000 (graphique 3, p.555). Cette décennie marque également la fin de la prééminence de l'histoire sociale et $\mathrm{du} \mathrm{XIX}^{\mathrm{e}}$ siècle dans les sections présentées, à la faveur notamment de l'histoire culturelle et de communications sur la théorie, la méthode ou la didactique de l'histoire et sur le second $\mathrm{XX}^{\mathrm{e}}$ siècle (graphiques 4 et 5 , p. 561). Le dernier congrès de la décennie, tenu à Francfort-sur-le-Main en 1998, a doublement marqué l'histoire de l'association (p. 723-734). Premièrement, il a été le théâtre d'un grand débat sur les historiens allemands durant le régime nationalsocialiste. Cette section a, dans les comptes rendus publics et dans la mémoire du congrès, éclipsé toutes les autres. Deuxièmement, c'est lors de ce congrès que les membres choisissent de renommer le nom de leur association en incluant explicitement les historiennes : il ne s'agit officiellement plus simplement du Verband des Historiker d'Allemagne, mais aussi des Historikerinnen.

Il est regrettable que les auteurs aient décidé d'arrêter leur ouvrage au tournant des $\mathrm{XX}^{\mathrm{e}}-\mathrm{XXI}^{\mathrm{e}}$ siècles et de ne pas prendre en compte les vingt dernières années. Choisir la rupture politique des années 1989/90 aurait pu être justifiée par les transformations liées à la réunification, la césure de l'an 2000 paraît par contre artificielle. On peut imaginer que les auteurs aient répugné à aborder la période la plus contemporaine, peut-être de peur d'être accusés de quitter le domaine historique. C'est une réelle perte pour l'ouvrage - certes, déjà très long - et pour les lectrices et lecteurs à la recherche d'une synthèse sur les évolutions les plus récentes de l'histoire universitaire allemande.

Histoire institutionnelle, histoire des sciences, histoire universitaire et histoire des hommes et des femmes qui ont porté ces institutions, mené ces recherches scientifiques, occupé ces chaires universitaires, cet ouvrage éclaire l'évolution de la discipline et de la profession historiques pendant plus d'un siècle. Les très larges questions que les auteurs se sont proposés de traiter conduisent à un livre particulièrement enrichissant sur le développement de la discipline historique en Allemagne et en Europe. L'ouvrage souffre parfois du trop-plein de détails, causé par le souci d'exhaustivité des auteurs qui se sont donnés pour objectif de retracer le plus finement possible l'histoire du Verband deutscher Historiker, de la Historiker-Gesellschaft et de leurs congrès respectifs. La division du livre en sous-parties claires et cohérentes, qui se font souvent écho de chapitre en chapitre, permet néanmoins aux lecteurs de consulter les thématiques qui les intéressent le plus. De ce point de vue, on ne peut qu'espérer, si ce n'est la traduction de l'ouvrage complet, au moins la publication d'un 
article résumant ses principales informations et conclusions, pour permettre au public historien français de mieux connaître l'histoire de leurs homologues allemands.

INDEX

Thèmes : Histoire des idées ; histoire sociale : histoire des sciences

Index chronologique : Époque contemporaine

\section{AUTEURS}

ANTONIN DUBOIS

EHESS 